\title{
Clinical Significance of Positive Surgical Margin after Radical Prostatectomy according to Pathological Stage
}

\author{
In Jae Lee, Jong Jin Oh, Tae Jin Kim, Byeong Do Song, Sangchul Lee, \\ Sung Kyu Hong, Sang Eun Lee, Seok-Soo Byun
}

Department of Urology, Seoul National University Bundang Hospital, Seongnam, Korea

Purpose: The purpose of this study was to investigate the positive surgical margin (PSM) as a predictive factor of biochemical recurrence $(\mathrm{BCR})$ in prostate cancer $(\mathrm{PCa})$ patients after radical prostatectomy $(\mathrm{RP})$ according to each pathological stage.

Materials and Methods: The records of 3,037 patients receiving RP were retrospectively reviewed. All patients were divided into 6 groups depending on pathological stage and presence of PSM. Cox proportional hazard analyses were performed to show the significance of PSM in all patients and in subgroup patients (T2, T3a, and T3b). The Kaplan-Meier analysis showed BCR-free survival rate of each group.

Results: Among total 3.307 patients, the mean age was 65.89 years and PSM rate was $18.7 \%$. During the 47.1 months, 550 patients had experienced BCR $(26.2 \%)$. According to groups, prostate-specific antigen, Gleason score, and BCR were significantly different. Ten-year BCR-free survival rate was $87.1 \%$ (T2R0), 65.9\% (T2R1), $60.1 \%$ (T3aR0), 43.0\% (T3aR1), 20.8\% (T3bR0), and 5.8\% (T3bR1). Each group had statistical differences with BCR-free survival except T2R1 and T3aR0 $(\mathrm{p}=0.090)$. PSM was significant in multivariate Cox analyses in total patients (hazard ratio, 2.091; 95\% confidence interval, 1.724-2.536; $p<0.001$ ) and in all subgroup with each stages.

Conclusions: PSM is a significant predictor to $\mathrm{BCR}$ after RP in all PCa patients and in each stage. T2R1 $\mathrm{PCa}$ had a similar BCR-free survival rate to T3RO patients during long-term follow-up, therefore careful management to T2R1 PCa as T3 should be necessary. (Korean J Urol Oncol 2016;14:159-164)

Key Words: Prostate neoplasms $\cdot$ Surgical margin

Received October 27, 2016, Revised December 5, 2016,

Accepted December 14, 2016

Corresponding Author: Seok-Soo Byun

Department of Urology, Seoul National University Bundang Hospital, 82 Gumi-ro 173beon-gil, Bundang-gu, Seongnam 13620, Korea

E-mail: ssbyun@snubh.org

Tel: +82-31-787-7342, Fax: +82-31-787-4057

- This work was supported by grant No. 02-2016-015 from the SNUBH (Seoul National University Bundang Hospital) Research Fund and supported by the Basic Science Research Program through the National Research Foundation of Korea (NRF), funded by the Ministry of Education, Science and Technology (NRF-2014R1A1A2059658).

\section{INTRODUCTION}

Prostate cancer was the 4 th most common cancer worldwide in 2012 and the second most common cancer diagnosed in men. ${ }^{1}$ Radical prostatectomy (RP) is most common treatment of prostate cancer. However, the prognostic outcome of patients with positive surgical margin (PSM) after RP is variable. Previous studies showed that PSM is not independently associated to the risk of biochemical recurrence (BCR). Ceylan et $\mathrm{al}^{2}$ reported that BCR occurred in $11.8 \%$ (11 of 93) of patients with PSM and in 45.9\% (17 of 37) of those without PSM (p

(i) (7) This is an Open Access article distributed under the terms of the Creative Commons Attribution Non-Commercial License (http://creativecommons.org/licenses/by-nc/4.0/) which permits unrestricted non-commercial use, distribution, and reproduction in any medium, provided the original work is properly cited. 2016 (C) Copyright The Korean Urological Oncology Society and The Korean Prostate Society. All Rights Reserved. 
$<0.001$ ). On the other hand, some studies suggested that in patients who underwent RP, PSM is a high risk factor of BCR, local recurrence, and distal metastasis. ${ }^{3,4}$ Furthermore, recent trials reported that PSM is a single predictor of BCR after $\mathrm{RP}^{5-7}$ Ohori et al. ${ }^{8}$ analyzed 5-year BCR-free survival rate of 478 patients who underwent RP mentioned that seminal vesicle invasion or lymph node metastases, PSMs had no effect on prognosis in patients with a high grade cancer. However, the data excluded association BCR with PSM according to pathological stage of patients after RP.

We found significant association between PSM and biochemical outcome in patients with $\mathrm{T} 2$ and $\mathrm{T} 3 \mathrm{a}$ prostate cancer underwent RP in a previous study. ${ }^{9}$ The data showed that men with T2 with PSM appear to have similar biochemical outcome compared with those with T3a tumor. But the impact of PSM on prognosis of prostate cancer after RP is not clear yet. ${ }^{2,3,10}$ In this present study, we examined the relationship between PSM and prognosis of patients who underwent RP with pathologic stage T2, T3a, and T3b at long-term follow-up.

\section{MATERIALS AND METHODS}

\section{Study Population}

After obtaining Institutional Review Board of Seoul National University Bundang Hospital (approval number: B-1612/375-103), 3,080 patients who underwent RP for prostate cancer at Seoul National University Bundang Hospital and postoperative follow-up for more than 2 years were enrolled in this study between November 2003 and September 2016. The participants were stratified into pathologic stage T2, T3a, and T3b. We excluded patients who underwent any other chemotherapy for prostate cancer. Also patients with lymph node invasion were excluded. In final, 3,037 men were included in our study.

\section{Study Group according to Pathological Stage and Presence of PSM}

Participants were divided into 6 groups depending on pathological findings: pT2R0, 1,807 (59.5\%) with T2 and NSM (negative surgical margin); pT2R1, 296 (9.7\%) with T2 and PSM; pT3aR0, 328 (10.8\%) with T3a and NSM; pT3aR1, 326(10.7\%) with T3a and PSM, pT3bR0: 100 (3.3\%) with T3b and NSM; pT3bR1, 180 (5.9\%) with T3b and PSM.

\section{Pathological Examinations}

The operative specimen analysis were similarly performed as previous study. ${ }^{9}$ Analyzed by single uropathologist, and sliced at 3- to 4-mm intervals on sagittal plane for evaluation of margin status parallel to the urethra. Every specimen was processed according to the Stanford protocol. Pathological features included Gleason classification, extracapsular extension (ECE), seminal vesicle invasion, and PSMs. ECE was defined as tumor piercing through the prostatic capsule and extending into the surrounding periprostatic tissue.

\section{Statistical Analyses}

The baseline characteristics included age, BMI, prostate-specific antigen (PSA) level at diagnosis, biopsy and pathological Gleason score (GS), clinical stage, prostate volume, surgical margin status, and follow-up PSA were compared with using the chi-square test, Fisher exact test, and the Mann-Whitney test. BCR was defined as two successive increases in PSA of $0.2 \mathrm{ng} / \mathrm{mL}$ or greater at least 2 months after RP. The BCR-free survival was estimated using the Kaplan-Meier survival technique and the log-rank test, which compared the BCR-free survival rate according to aforementioned groups. Multivariate Cox proportional hazard analysis was done to identify various variables between BCR-free survival among total patients, T2 patients, T3a patients and T3b patients. All statistical tests were 2 -sided with a significance level defined as $\mathrm{p} \leq 0.05$ and performed using the SPSS ver. 15.0 (SPSS Inc., Chicago, IL, USA).

\section{RESULTS}

Among total patients, mean age \pm standard deviation was $65.89 \pm 6.81$ years. Overall rate of PSM was $18.7 \%$, seminal vesicle invasion was $9.7 \%$ and ECE was $30.3 \%$. During the median follow-up was 47.1 months, total BCR rate was $18.7 \%$.

In pT2R0, patients with pathological T2 and NSM $(n=1,807)$, 103 patients had BCR (5.7\%). In pT2R1 ( $\mathrm{n}=296), 65$ patients recurred biochemically (21.9\%). Patients with BCR was 69 in pT3aR0 (21.0\%), 143 in pT3aR1 $(43.8 \%), 43$ in pT3bR0 (43\%), and 127 in pT3bR1 (70.5\%). Major pathological GS were reported GS 7 in pathological stage T2 and T3a. T3b, including pT3bR0 and pT3bR1, had higher portion of GS 8 or more, but GS 8 or more was not major in pT3bR0 (37\%, GS 
Table 1. Clinicopathological baselines of patients

\begin{tabular}{|c|c|c|c|c|c|c|c|}
\hline Variable & pT2R0 & pT2R1 & pT3aR0 & pT3aR1 & pT3bR0 & pT3bR1 & p-value \\
\hline No. of patients (\%) & $1,807(59.5)$ & $296(9.7)$ & $328(10.8)$ & $326(10.7)$ & $100(3.3)$ & $180(5.9)$ & \\
\hline Age (y) & $65.51 \pm 6.98$ & $65.46 \pm 6.77$ & $67.12 \pm 6.19$ & $66.45 \pm 6.52$ & $66.89 \pm 6.40$ & $67.00 \pm 6.52$ & 0.112 \\
\hline BMI $\left(\mathrm{kg} / \mathrm{m}^{2}\right)$ & $24.38 \pm 6.31$ & $24.47 \pm 2.83$ & $25.17 \pm 13.13$ & $24.39 \pm 2.68$ & $24.38 \pm 2.42$ & $24.72 \pm 2.58$ & 0.521 \\
\hline PSA (ng/mL) & & & & & & & $<0.001$ \\
\hline Median & 6.70 & 8.20 & 10.13 & 12.00 & 12.88 & 26.00 & \\
\hline Mean \pm SD & $8.84 \pm 9.67$ & $10.99 \pm 10.14$ & $13.47 \pm 10.89$ & $17.65 \pm 19.02$ & $21.65 \pm 22.84$ & $34.13 \pm 31.88$ & \\
\hline Biopsy Gleason score & & & & & & & $<0.001$ \\
\hline $4-5$ & $5(0.3)$ & $0(0)$ & $1(0.3)$ & $0(0)$ & $0(0)$ & $0(0)$ & \\
\hline 6 & $946(52.3)$ & 147 (49.6) & $71(21.6)$ & $84(25.7)$ & $12(12)$ & $4(2.2)$ & \\
\hline 7 & $682(37.7)$ & $115(38.8)$ & $165(50.3)$ & $166(50.9)$ & $54(54)$ & $64(35.6)$ & \\
\hline $8^{-10}$ & $161(8.9)$ & $34(11.4)$ & $90(27.4)$ & $74(22.7)$ & $32(32)$ & $113(62.8)$ & \\
\hline Clinical stage & & & & & & & 0.331 \\
\hline $\mathrm{T} 1$ & $1,230(68.1)$ & $179(60.5)$ & $156(47.6)$ & $144(44.2)$ & $40(40)$ & $42(23.3)$ & \\
\hline $\mathrm{T} 2$ & $512(28.3)$ & $101(34.1)$ & $125(38.1)$ & $134(41.1)$ & $35(35)$ & $42(23.3)$ & \\
\hline $\mathrm{T} 3$ & $65(3.6)$ & $16(2)$ & $47(14.3)$ & $48(14.7)$ & $25(25)$ & $97(53.9)$ & \\
\hline Pathological Gleason s & ore & & & & & & $<0.001$ \\
\hline 6 & $296(16.4)$ & $26(8.8)$ & $4(1.2)$ & $6(1.8)$ & $0(0)$ & $0(0)$ & \\
\hline 7 & $1,427(78.9)$ & $247(83.4)$ & $263(80.2)$ & $254(77.9)$ & $63(63)$ & $72(40)$ & \\
\hline $8-10$ & $84(4.6)$ & $23(7.8)$ & $61(18.6)$ & $66(20.2)$ & $37(37)$ & $108(60)$ & \\
\hline Prostate volume $(\mathrm{mL})$ & $38.44 \pm 15.82$ & $35.44 \pm 15.14$ & $33.86 \pm 12.09$ & $35.01 \pm 16.48$ & $34.71 \pm 13.71$ & $38.24 \pm 16.24$ & $<0.001$ \\
\hline BCR & $103(5.7)$ & $65(21.9)$ & $69(21.0)$ & $143(43.9)$ & $43(43)$ & $127(70.6)$ & $<0.001$ \\
\hline
\end{tabular}

Values are presented as number (\%) or mean \pm standard deviation unless otherwise indicated.

BMI: body mass index, PSA: prostate-specific antigen, SD: standard deviation, BCR: biochemical recurrence.

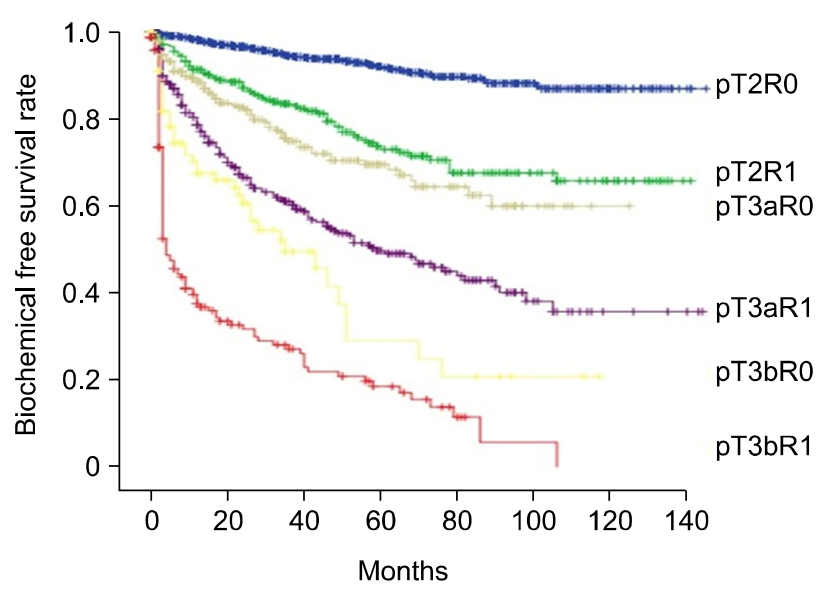

Fig. 1. Biochemical recurrence-free survivals according to surgical marginal status and stage among the overall patients.

7: 63\%). Further details and characteristics are on Table 1.

In 2103 patients without ECE, 296 had PSM (14.1\%). Groups with stage T3a had 326 men with PSM (49.8\%). In addition, 90 (58.8\%) had PSM among 153 patients with stage T3b.

Of 6 groups, there was no significant difference in mean age,
BMI, and clinical stage. However patients with high pathological stage or PSM had higher preoperative PSA value, higher biopsy and pathological GS, and higher BCR rate than patients with low pathological stage or NSM $(\mathrm{p}<0.001)$. Details of characteristics were shown in Table 1.

In this study, 550 patients had BCR (26.2\%). As shown in Fig. 1, Ten-year BCR-free survival rate was $87.1 \%$ (T2R0), $65.9 \%$ (T2R1), $60.1 \%$ (T3aR0), $43.0 \%$ (T3aR1), $20.8 \%$ (T3bR0), and 5.8\% (T3bR1). Each groups had statistically differences at BCR-free survival except the T2R1 and T3aR0 comparison $(p=0.090)$. Each comparison among groups was shown in Table 2.

In multivariate Cox proportional hazard analysis among total patients, preoperative PSA, pathological GS and pathological stage were performed to independent factors of BCR at multivariate analysis $(\mathrm{p}<0001)$ (Table 3$)$. Other factors such as age and prostate volume had no significant relation with BCR. Also variables were analyzed according to pathological stage for investigate effect to BCR-free survival. Preoperative PSA and pathological GS were significant predictors for BCR in all groups ( $\mathrm{p}<0.001$; GS with T3b, $\mathrm{p}=0.002$ ). Nevertheless, PSA 
Table 2. Log-rank test according to BCR rates between each group and the other groups

\begin{tabular}{|c|c|c|c|c|c|c|c|c|c|c|c|c|}
\hline \multirow{2}{*}{$\begin{array}{l}\text { Pathological } \\
\text { stage }\end{array}$} & \multicolumn{2}{|c|}{ pT2R0 } & \multicolumn{2}{|c|}{ pT2R1 } & \multicolumn{2}{|c|}{ pT3aR0 } & \multicolumn{2}{|c|}{ pT3aR1 } & \multicolumn{2}{|c|}{ pT3bR0 } & \multicolumn{2}{|c|}{ pT3bR1 } \\
\hline & OR & p-value & OR & p-value & OR & p-value & OR & p-value & OR & p-value & OR & p-value \\
\hline pT2R0 & - & - & 69.06 & $<0.001$ & 123.94 & $<0.001$ & 407.44 & $<0.001$ & 360.14 & $<0.001$ & $1,240.52$ & $<0.001$ \\
\hline $\mathrm{pT} 2 \mathrm{R} 1$ & 69.06 & $<0.001$ & - & - & 2.87 & 0.090 & 39.17 & $<0.001$ & 53.23 & $<0.001$ & 204.16 & $<0.001$ \\
\hline pT3aR0 & 123.94 & $<0.001$ & 2.87 & 0.090 & - & - & 18.01 & $<0.001$ & 30.58 & $<0.001$ & 162.69 & $<0.001$ \\
\hline pT3aR1 & 407.44 & $<0.001$ & 39.17 & $<0.001$ & 18.01 & $<0.001$ & - & - & 5.73 & 0.017 & 92.44 & $<0.001$ \\
\hline pT3bR0 & 360.14 & $<0.001$ & 53.23 & $<0.001$ & 30.58 & $<0.001$ & 5.73 & 0.017 & - & - & 18.03 & $<0.001$ \\
\hline pT3bR1 & $1,240.52$ & $<0.001$ & 204.16 & $<0.001$ & 162.69 & $<0.001$ & 92.44 & $<0.001$ & 18.03 & $<0.001$ & - & - \\
\hline
\end{tabular}

BCR: biochemical recurrence, OR: odds ratio.

Table 3. Multivariate Cox regression models for the prediction of biochemical recurrence-free survival after radical prostatectomy according to pathologic stage and among all patients

\begin{tabular}{|c|c|c|c|}
\hline Variable & Hazard ratio & $95 \% \mathrm{CI}$ & p-value \\
\hline \multicolumn{4}{|c|}{ Multivariate Cox regression analysis of total patients } \\
\hline Age & 1.000 & $0.987-1.012$ & 0.966 \\
\hline Prostate volume & 0.998 & $0.992-1.004$ & 0.457 \\
\hline Preoperative PSA & 1.008 & $1.005-1.011$ & $<0.001$ \\
\hline Pathologic Gleason score & 3.723 & $2.118-6.544$ & $<0.001$ \\
\hline Positive surgical margin & 2.091 & $1.724-2.536$ & $<0.001$ \\
\hline Extracapsular extension & 2.838 & $2.289-3.520$ & $<0.001$ \\
\hline Seminal vesicle invasion & 2.909 & $2.363-3.580$ & $<0.001$ \\
\hline \multicolumn{4}{|c|}{ Multivariate Cox regression analysis of 2,103 patients with pathological stage T2 } \\
\hline Age & 0.978 & $0.956-1.000$ & 0.054 \\
\hline Prostate volume & 0.995 & $0.984-1.006$ & 0.339 \\
\hline Preoperative PSA & 1.022 & $1.016-1.028$ & $<0.001$ \\
\hline Pathologic Gleason score & 4.294 & $2.242-8.223$ & $<0.001$ \\
\hline Positive surgical margin & 2.942 & $2.144-4.036$ & $<0.001$ \\
\hline \multicolumn{4}{|c|}{ Multivariate Cox regression analysis of 654 patients with pathological stage T3a } \\
\hline Age & 0.998 & $0.984-1.011$ & 0.733 \\
\hline Prostate volume & 0.995 & $0.989-1.001$ & 0.094 \\
\hline Preoperative PSA & 1.017 & $1.015^{-1.020}$ & $<0.001$ \\
\hline Pathologic Gleason score & 5.478 & $3.140-9.559$ & $<0.001$ \\
\hline Positive surgical margin & 3.580 & $3.003-4.269$ & $<0.001$ \\
\hline \multicolumn{4}{|c|}{ Multivariate Cox regression analysis of 280 patients with pathological stage T3b } \\
\hline Age & 1.006 & $0.982-1.031$ & 0.628 \\
\hline Prostate volume & 1.004 & $0.994-1.013$ & 0.446 \\
\hline Preoperative PSA & 1.003 & $0.998-1.007$ & 0.255 \\
\hline Pathologic Gleason score & 1.745 & $1.221-2.494$ & 0.002 \\
\hline Positive surgical margin & 1.774 & $1.291-2.436$ & $<0.001$ \\
\hline
\end{tabular}

CI: confidence interval, PSA: prostate-specific antigen.

did not have effect on BCR-free survival in 280 patients with T3b (pT3bR0 and pT3bR1) on multivariate cox analysis (hazard ratio [HR], 0.995; $\mathrm{p}=0.094$ ).

PSM was thought to significant effect on biochemical outcomes such as preoperative PSA, pathological GS and pathological stage in multivariate Cox analysis (HR, 2.091; $\mathrm{p}<$ 0.001 ). This result was not changed whether pathologic status change or not. In patients with T2, PSM showed that risk of BCR was higher than NSM almost three times (HR, 2.942; 95\% CI, 2.144-4.036; $\mathrm{p}<0.001)$. This tendency more pronounced in stage T3a (HR, 2.580; CI, 3.003-4.269; $\mathrm{p}<0.001)$. 


\section{DISCUSSION}

ECE is known predictor related with elevate risk of biochemical recurrence in patients underwent RP. ${ }^{4,11}$ But PSM influence on prognosis of prostate cancer after RP remains controversial. In the current study, surgical margin state was acted on as a significant predictor of BCR in patients who underwent RP. Ploussard et al. ${ }^{5}$ showed that PSM was significantly predictive for $\mathrm{BCR}(\mathrm{p}<0.001 ; \mathrm{HR}, 2.6)$ and the 5 -year RFS was $84.4 \%$ in men with negative margins compared to $57.5 \%$ in the case of PSM. Furthermore, BCR for patients with close surgical margin $(<1 \mathrm{~mm})$ was tantamount to PSM patients. ${ }^{6}$ On the other hand, some studies revealed that PSMs were associated with BCR but not long-term oncologic outcomes ${ }^{10}$ or margin status was not an important factor of biochemical recurrence different to PSA and GS. ${ }^{3}$ There were conflicting studies that showed that the prognosis of patients with PSM without ECE after RP is not significantly different from that of patients with cancers confined to the prostate. ${ }^{3}$

Furthermore, a study showed that patients with PSM without ECE have no statistical difference as compared with patients with NSM without ECE. ${ }^{12}$ Nevertheless, the same group reported contradictory finding in later study. ${ }^{7}$ Kausik et al. ${ }^{13}$ also reported that the overall prognosis of patients with extraprostatic disease in the absence of surgical margin involvement is excellent and comparable to that of patients with organ-confined disease. The study including 1,621 patients from the SEARCH Database reported that patients with PSM without ECE had same BCR rate to patients with NSM with ECE. Moreover, it showed that patients with ECE had similar BCR rates irrespective of margin status. ${ }^{14}$ This result imply that the prostate surgical margin status is not an important predictor of BCR. In these patients, ECE outweighed in importance by surgical margin status for predicting BCR. Such result may have important surgical implications. If surgical margin status is not important in PSA outcomes, wide excision of the neurovascular bundle or other periprostatic tissue in an attempt to prevent PSM may be ineffective on biochemical outcomes.

In our study, PSM was influenced to BCR rate of patients with pathologic stages $\mathrm{T} 2$ and $\mathrm{T} 3$ prostate cancer, which were significantly higher in men with T2 and PSM. In a recent study done by Budäus et al. ${ }^{15}$, 4,490 patients in single institution participated between 1992 and 2008, descripted that PSM is hav- ing outstandingly adverse effect on prognosis after RP. This result supports our findings.

Previous studies showed that surgical margin status was not associated with prognosis of patients underwent RP. Ohori et al. ${ }^{8}$ showed that surgical margin status was no effective factor to worse prognosis in several patients such as men with lymph node positive, ECE, seminal vesicle invasion and high grade cancer $(\mathrm{GS} \geq 7){ }^{8}$ Other studies mentioned that surgical margin status may not be prominent factor of PSA outcome in patients with ECE. ${ }^{14}$ These results may have important surgical implications. In localized prostate cancer, nerve-sparing RP was no influence to margin status. ${ }^{16}$ If PSM have no importance about prognosis in high stage prostate cancer, wide surgical excision of the neurovascular bundle or other periprostatic tissue may not be a necessary procedure. However, in our current study, patients with PSM were observed to have worse BCR rates than groups with NSM and same pathological stage. This result can be missing ECM from incomplete pathological evaluation. However, it is difficult to think that microscopic ECE would be missed large number of men with a PSM and no seminal vesicle invasion.

Our data suggested that men with PSM may be receiving adjuvant therapy (aRT) after surgery. There is a study done to compare the effect of early salvage radiation therapy (esRT) compared to long-term aRT. ${ }^{17}$ In 510 pT3pN0 patients with undetectable PSA after RP, 243 patients (48\%) underwent aRT, and 267 (52\%) underwent initial observation. Within the latter group, 141 patients experienced PSA relapse and received esRT. Their results suggested that no significant differences between of esRT to long term aRT for T3p patients, with no margin status. ${ }^{17}$ However, men with PSM without ECE have similar BCR rates those with NSM with ECE in our data. This result suggests that aRT can be having same advantage in prognosis of both groups.

There are several limitations of our study that should be considered, including its retrospective nature. In second place, this study included many surgeons; surgical techniques may be not having been equal in every RP. Margin status can be influenced by technical errors like capsular injury or cancer status which complete resection is impossible due to wide invasion. Surgical artifacts such as crush, thermal cautery or electrocautery are affected to pathological interpretation because of lack of a clearly definable prostatic capsule along the edge of the gland. ${ }^{18}$ Also we could not analyzed location and length of PSM. The influ- 
ence of margin locations and length are controversial. Finally, the median follow-up of our study is 47 months, we cannot evaluate more long-term period outcomes consequently. Given the long natural history of prostate cancer, longer follow-up durations are needed to confirm our present findings.

\section{CONCLUSIONS}

In our study, we found that PSM is significantly related with biochemical recurrence in pathological stage T2 and T3 prostate cancer underwent RP. Furthermore, T2 prostate cancers with PSM have shown similar BCR survival to patients with pathologic stage T3a and NSM. These results suggest that PSM should be considered in planning treatment for patients after RP.

This study will deliver more data in the future and thus be able to address some of these important issues using a global perspective.

\section{CONFLICT OF INTEREST}

The authors claim no conflicts of interest.

\section{REFERENCES}

1. Lee MS, Cho JY, Kim SY, Cheon GJ, Moon MH, Oh S, et al. Diagnostic value of integrated PET/MRI for detection and localization of prostate cancer: Comparative study of multiparametric MRI and PET/CT. J Magn Reson Imaging 2016 Sep 1 [Epub]. https://doi.org/10.1002/jmri.25384

2. Ceylan C, Tonyali S, Keles I. Impact of positive surgical margin on biochemical recurrence following radical prostatectomy in locally advanced prostate cancer. Kaohsiung J Med Sci 2016;32:514-7

3. Stamey TA, McNeal JE, Yemoto CM, Sigal BM, Johnstone IM. Biological determinants of cancer progression in men with prostate cancer. JAMA 1999;281:1395-400.

4. Blute ML, Bostwick DG, Seay TM, Martin SK, Slezak JM, Bergstralh EJ, et al. Pathologic classification of prostate carcinoma: the impact of margin status. Cancer 1998;82:902-8

5. Ploussard G, Agamy MA, Alenda O, Allory Y, Mouracade $\mathrm{P}$, Vordos $\mathrm{D}$, et al. Impact of positive surgical margins on prostate-specific antigen failure after radical prostatectomy in adjuvant treatment-naïve patients. BJU Int 2011;107:1748-54

6. Whalen MJ, Shapiro EY, Rothberg MB, Turk AT, Woldu SL, Roy Choudhury A, et al. Close surgical margins after radical prostatectomy mimic biochemical recurrence rates of positive margins. Urol Oncol 2015;33:494.e9-494.e14

7. Chuang AY, Nielsen ME, Hernandez DJ, Walsh PC, Epstein JI. The significance of positive surgical margin in areas of capsular incision in otherwise organ confined disease at radical prostatectomy. J Urol 2007;178(4 Pt 1):1306-10

8. Ohori M, Wheeler TM, Kattan MW, Goto Y, Scardino PT. Prognostic significance of positive surgical margins in radical prostatectomy specimens. J Urol 1995;154:1818-24

9. Oh JJ, Hong SK, Byun SS, Choe G, Lee SE. Prognostic significance of positive surgical margins after radical prostatectomy among pT2 and pT3a prostate cancer. Urol Oncol 2013;31:595-600

10. Wadhwa H, Terris MK, Aronson WJ, Kane CJ, Amling CL, Cooperberg MR, et al. Long-term oncological outcomes of apical positive surgical margins at radical prostatectomy in the Shared Equal Access Regional Cancer Hospital cohort. Prostate Cancer Prostatic Dis 2016;19:423-8

11. Cheng L, Darson MF, Bergstralh EJ, Slezak J, Myers RP, Bostwick DG. Correlation of margin status and extraprostatic extension with progression of prostate carcinoma. Cancer 1999;86:1775-82

12. Barocas DA, Han M, Epstein JI, Chan DY, Trock BJ, Walsh $\mathrm{PC}$, et al. Does capsular incision at radical retropubic prostatectomy affect disease-free survival in otherwise organ-confined prostate cancer? Urology 2001;58:746-51

13. Kausik SJ, Blute ML, Sebo TJ, Leibovich BC, Bergstralh EJ, Slezak J, et al. Prognostic significance of positive surgical margins in patients with extraprostatic carcinoma after radical prostatectomy. Cancer 2002;95:1215-9

14. Freedland SJ, Aronson W, Presti JC Jr, Kane CJ, Terris MK, Elashoff D, et al. Should a positive surgical margin following radical prostatectomy be pathological stage $\mathrm{T} 2$ or T3? Results from the SEARCH database. J Urol 2003;169:2142-6

15. Budäus L, Isbarn H, Eichelberg C, Lughezzani G, Sun M, Perrotte $\mathrm{P}$, et al. Biochemical recurrence after radical prostatectomy: multiplicative interaction between surgical margin status and pathological stage. J Urol 2010;184:1341-6

16. Alkhateeb SS, Alibhai SM, Finelli A, Fleshner NE, Jewett MA, Zlotta AR, et al. Does nerve-sparing radical prostatectomy increase the risk of positive surgical margins and biochemical progression? Urol Ann 2010;2:58-62

17. Fossati N, Karnes RJ, Boorjian SA, Moschini M, Morlacco A, Bossi A, et al. Long-term impact of adjuvant versus early salvage radiation therapy in pT3N0 prostate cancer patients treated with radical prostatectomy: results from a multi-institutional series. Eur Urol 2016 Jul 30 [Epub]. https://doi.org/10.1016/j.eururo.2016.07.028

18. Evans AJ, Henry PC, Van der Kwast TH, Tkachuk DC, Watson K, Lockwood GA, et al. Interobserver variability between expert urologic pathologists for extraprostatic extension and surgical margin status in radical prostatectomy specimens. Am J Surg Pathol 2008;32:1503-12 